\title{
TFEB-mediated lysosomal biogenesis and lysosomal drug sequestration confer resistance to MEK inhibition in pancreatic cancer
}

Ben Zhao 1,2, Laura Dierichs ${ }^{1,2}$, Jiang-Ning Gu ${ }^{1,2,3}$, Marija Trajkovic-Arsic ${ }^{1,2}$, Ralf Axel Hilger ${ }^{4}$, Konstantinos Savvatakis ${ }^{1,2}$, Silvia Vega-Rubin-de-Celis ${ }^{5}$, Sven-Thorsten Liffers ${ }^{1,2}$, Samuel Peña-Llopis ${ }^{1,2,6}$, Diana Behrens ${ }^{7}$, Stephan Hahn ${ }^{8}$, Jens T. Siveke (10), and Smiths S. Lueong (10),

\begin{abstract}
Oncogenic KRAS mutations are encountered in more than $90 \%$ of pancreatic ductal adenocarcinomas. MEK inhibition has failed to procure any clinical benefits in mutant RAS-driven cancers including pancreatic ductal adenocarcinoma (PDAC). To identify potential resistance mechanisms underlying MEK inhibitor (MEKi) resistance in PDAC, we investigated lysosomal drug accumulation in PDAC models both in vitro and in vivo. Mouse PDAC models and human PDAC cell lines as well as human PDAC xenografts treated with the MEK inhibitor trametinib or refametinib led to an enhanced expression of lysosomal markers and enrichment of lysosomal gene sets. A time-dependent, increase in lysosomal content was observed upon MEK inhibition. Strikingly, there was a strong activation of lysosomal biogenesis in cell lines of the classical compared to the basal-like molecular subtype. Increase in lysosomal content was associated with nuclear translocation of the Transcription Factor EB (TFEB) and upregulation of TFEB target genes. siRNA-mediated depletion of TFEB led to a decreased lysosomal biogenesis upon MEK inhibition and potentiated sensitivity. Using LCMS, we show accumulation of MEKi in the lysosomes of treated cells. Therefore, MEK inhibition triggers lysosomal biogenesis and subsequent drug sequestration. Combined targeting of MEK and lysosomal function may improve sensitivity to MEK inhibition in PDAC.
\end{abstract}

\section{Introduction}

Pancreatic ductal adenocarcinoma (PDAC) is among the most aggressive and lethal malignant human diseases, with a 5 -year survival rate of less than $8 \%^{1}$. Given the high frequency of tumors diagnosed at an advanced stage and the high rate of intrinsic and acquired resistance to virtually any therapy, blocking resistance mechanisms

\footnotetext{
Correspondence: Jens T. Siveke (j.siveke@dkfz.de)

'Division of Solid Tumor Translational Oncology, German Cancer Consortium (DKTK, partner site Essen) and German Cancer Research Center, DKFZ, Heidelberg, Germany, Division of Solid Tumor Translational Oncology, German Cancer Consortium (DKTK, partner site Essen) and German Cancer Research Center, DKFZ, Heidelberg, Germany, Essen, Germany

${ }^{2}$ Institute for Developmental Cancer Therapeutics, West German Cancer Center, University Hospital Essen, Essen, Germany, Heidelberg, Germany

Full list of author information is available at the end of the article.

These authors contributed equally: Jens T. Siveke, Smiths S. Lueong

Edited by I. Amelio
}

remains a key challenge. Recent evidence points to the existence of at least two major molecular subtypes: the quasi-mesenchymal or basal-like and the classical subtypes, with differences in signaling activities and therapy response $^{2,3}$.

Activating mutations in KRAS is the most frequent genetic alteration observed in more than $90 \%$ of PDAC tumors ${ }^{4}$. These mutations promote proliferation and inhibit apoptosis via the RAF/MEK/ERK and PI3K/AKT pathway ${ }^{5-7}$, thereby making Ras inhibition an attractive drug target. Unfortunately, there are currently no effective therapies for about $30 \%$ of all Ras mutant human malignancies ${ }^{8}$. This is in part due to lack of target specificity and feedback loops, which have rendered direct KRAS targeting very challenging and efforts have been focused on targeting downstream signaling pathways such as

\section{(-) The Author(s) 2020}

(c) (i) Open Access This article is licensed under a Creative Commons Attribution 4.0 International License, which permits use, sharing, adaptation, distribution and reproduction cc. in any medium or format, as long as you give appropriate credit to the original author(s) and the source, provide a link to the Creative Commons license, and indicate if changes were made. The images or other third party material in this article are included in the article's Creative Commons license, unless indicated otherwise in a credit line to the material. If material is not included in the article's Creative Commons license and your intended use is not permitted by statutory regulation or exceeds the permitted use, you will need to obtain permission directly from the copyright holder. To view a copy of this license, visit http://creativecommons.org/licenses/by/4.0/. 
MEK/ERK ${ }^{7,9}$. Despite development of highly-specific MEK inhibitors and good on-target efficacy, MEK inhibition has unfortunately failed to show any clinical benefit ${ }^{10,11}$ in PDAC as well as in other cancer entities ${ }^{12,13}$. The mechanisms underlying the inefficacy of MEK inhibition in PDAC are still not well understood. However, combinatorial strategies may constitute promising avenues, as recent combination of MEK and SHP2 inhibition have shown to overcome RTK-mediated pathway reactivation in KRAS-mutant tumors ${ }^{14,15}$.

Hydrophobic anticancer agents with weak base properties are easily trapped in the acidic medium of the lysosomes by cation trapping ${ }^{16}$. Such organelle-mediated drug sequestration keeps drugs far from their molecular targets, thereby inducing drug resistance ${ }^{17}$. Additionally, the number of drug-containing lysosomes per cell has been shown to be directly correlated with the extent of resistance $^{17}$ and lysosomal drug accumulation is known to trigger cytoplasmic to nuclear translocation of the transcription factor EB (TFEB). TFEB is a master regulator of lysosomal biogenesis and autophagy, dependent on the mechanistic target of rapamycin complex $1(\text { mTORC } 1)^{17,18}$. Lysosomal drug sequestration also induces lysosomal exocytosis and hence drug excretion from within target cells ${ }^{16}$. Investigating the role of lysosomal drug sequestration upon MEK inhibition in PDAC is therefore warranted. This is further strengthened by recent reports, where MEK inhibition is reported to elicit protective autophagy in Ras-driven cancer ${ }^{19}$.

meanwhile pathway reactivation and reliance on interferon-mediated signaling has been reported ${ }^{20,21}$, the role of lysosomal drug sequestration of MEK inhibitors in PDAC has not been addressed. We herein report on lysosomal sequestration of MEK inhibitors by PDAC cells in vitro and in vivo and a strong activation of lysosomal biogenesis upon MEKi treatment. Furthermore, we show that disruption of lysosomal biogenesis by TFEB knockdown partially sensitizes PDAC cells to trametinib treatment and demonstrate the presence of trametinib in lysosomes.

\section{Results}

\section{MEKi treatment triggers expression of lysosome-} associated genes in mouse PDAC models

Using mouse PDAC-derived cell lines and mouse PDAC models, we investigated the impact of MEK inhibition on lysosomal biogenesis. Cell lines from mouse PDAC models were treated with trametinib for a period of $48 \mathrm{~h}$ with single dose $\mathrm{IC}_{50}$ and trametinib-resistant mouse PDAC cell lines were generated by chronic exposure. Gene expression and gene set enrichment analyses (GSEA) comparing short-term or trametinib-resistant cells with vehicle control revealed an increase in the expression of lysosomal markers as well as lysosomal enzymes such as MCOLN1, LAMP1, LAMP2, ATP6VOD1 $A T P 6 V 1 H$ (Fig. 1a, b). Immunostaining of the lysosomal marker $L A M P 1$ was performed on tumor sections from short-term refametinib-treated mouse PDAC tumors equally showed an overexpression of the LAMP1 gene product upon MEKi treatment (Fig. 1c).

In summary, MEK inhibition with distinct small molecule inhibitors led to increased lysosomal biogenesis signatures in various in vitro and in vivo models of murine PDAC.

\section{MEKi-induced lysosomal biogenesis is time-dependent}

We next asked whether lysosomal biogenesis upon exposure to MEK inhibition is dose- and/or timedependent. Exposure of human PDAC cell lines to single $\mathrm{IC}_{50}$ doses of trametinib for different time periods revealed a linear time-dependency in lysosomal levels, up to $72 \mathrm{~h}$ post-treatment (Fig. 2a). However, exposure of the same cell lines to increasing doses (from $0.25 \mathrm{x} \mathrm{IC}_{50}$ to $2 \mathrm{x}$ $\mathrm{IC}_{50}$ ) of trametinib did not show any trend (Fig. 2b), indicating that drug concentration is less relevant for firing transcriptional programs responsible for increased lysosomal biogenesis. Given that PDAC constitute a heterogeneous disease with at least two major subtypes described, each having significant differences in therapy response and disease prognosis, we characterized our cell lines using the mesenchymal cell marker vimentin and the epithelial cell marker E-cadherin. All five epithelial human PDAC cell lines further used in the study had low vimentin expression and higher E-cadherin expression, meanwhile all mesenchymal cells had a higher vimentin expression and a lower E-cadherin expression (Fig. 2c). Cells were classified either as being of the classical or quasi-mesenchymal subtype based on previous classification $^{22}$. All further analyses in this study were based on these cell lines.

\section{MEKi increases the lysosomal content and induces nuclear translocation of TFEB}

We next investigated the association between the observed increase in the expression of lysosomalassociated genes and cellular lysosomal levels. Using a fluorescent lysosome-specific dye, lysosomal content was measured by flow cytometry. As shown in Fig. 3a, cellular lysosomal levels were significantly increased in the trametinib-treated group compared with the vehicle control and medium-only groups. Unexpectedly, there was a stronger increase in lysosomal levels in PDAC cell lines related to the classical or epithelial (cl) subtype (HPAC, Hup-T4, Patu8988S, HPAF-II) compared with quasimesenchymal or basal-like (qm) PDAC cell lines (MiaPaCa-2, PSN-1, Patu8988T, KP4 and Panc-1) upon MEKi treatment (Fig. 3a). Upon trametinib treatment, an increase in the protein (Fig. 3b) and transcript (Fig. 2c) 


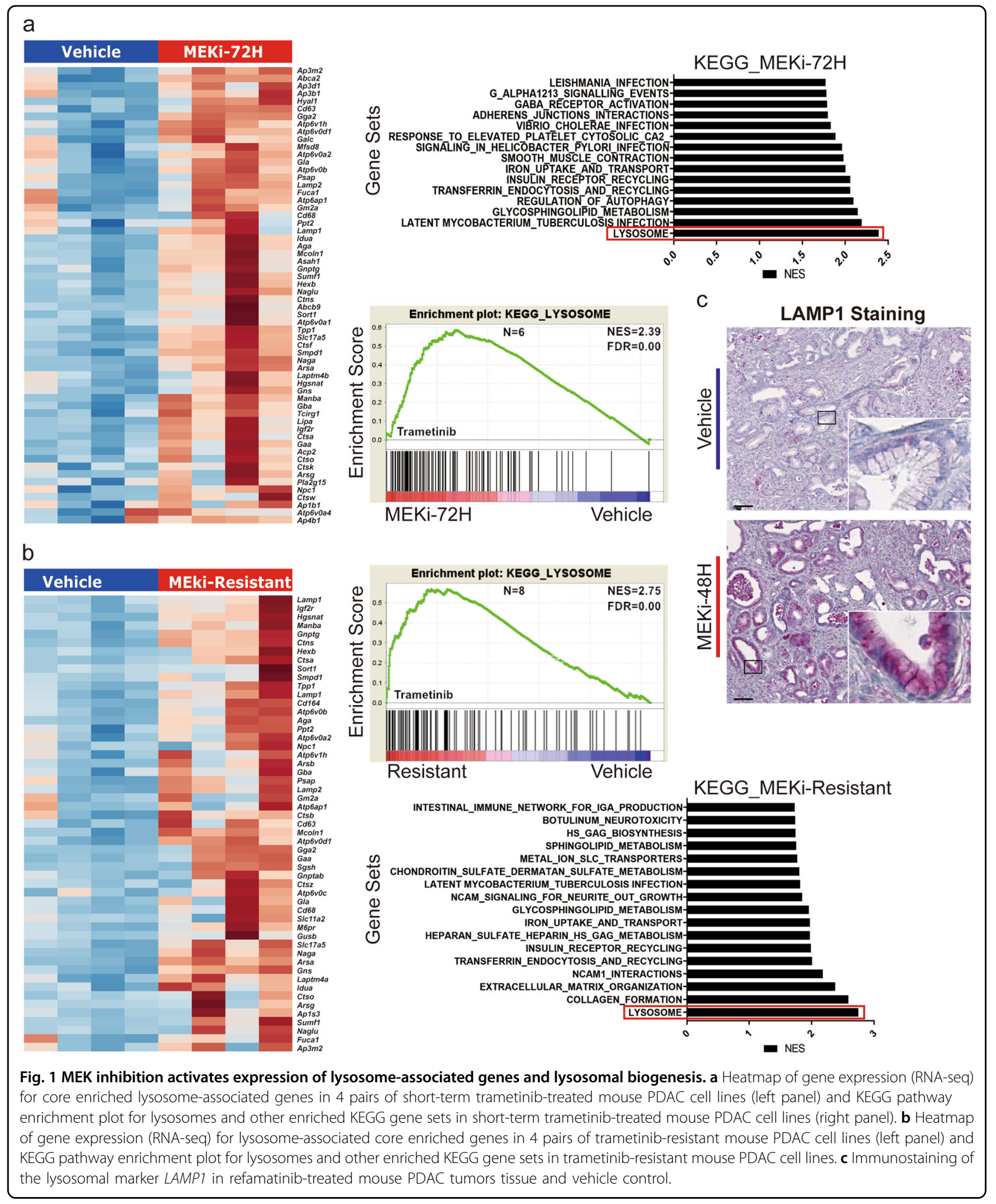

expression of lysosomal markers $L A M P 1$ and $L A M P 2$ as well as the lysosomal hydrolase CTSD. Furthermore, significant increases in the expression of direct TFEB targets such as ATPV1A, ATPVOD1 (Fig. 3d, e and Supplementary Fig. 2) as well as other lysosomal ATPases and TFEB direct target genes such as ATP6VOC, ATP6V1B2 and CREG1 


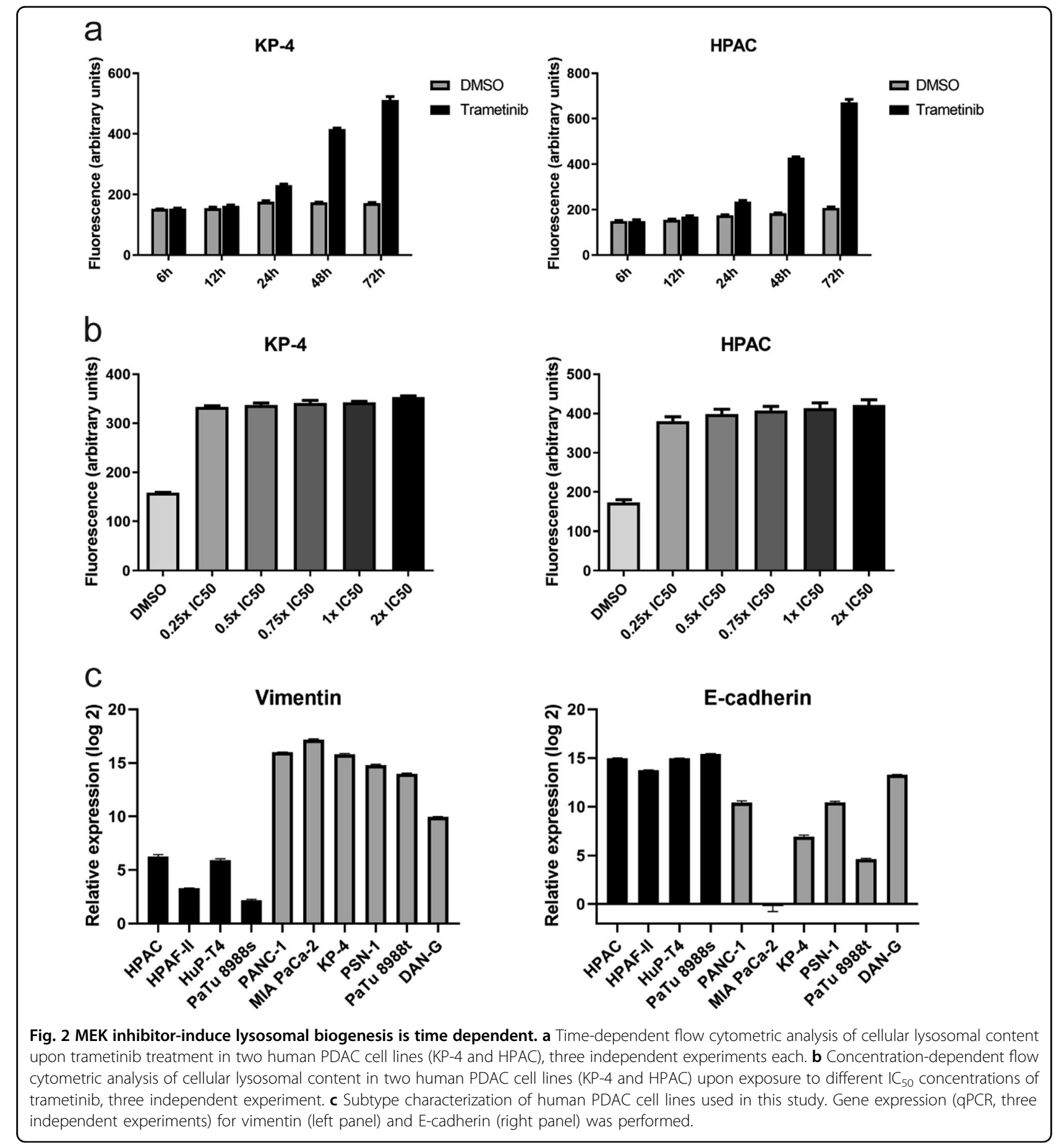

(Supplementary Fig. 1a). Concomitantly, increase in cellular lysosomal levels were associated with cytoplasmic to nuclear translocation of TFEB as revealed by immunofluorescence staining (Fig. $3 \mathrm{f}$ and Supplementary Figs. 3 and 4). In the qm PDAC subtype, nuclear TFEB was present in the vehicle control group and increased upon treatment, as opposed to the cl subtype, where TFEB was exclusively cytoplasmic in the vehicle control group.

\section{TFEB silencing inhibits Trametinib-induced lysosomal} biogenesis

To investigate the association between MEKi-induced lysosomal biogenesis and nuclear translocation of TFEB, we performed siRNA-mediated TFEB silencing. Four siRNA contructs targeting the TFEB locus (Supplementary Table 1) were evaluated and the best performing construct selected (Supplementary Fig. 4). Using the best-performing 


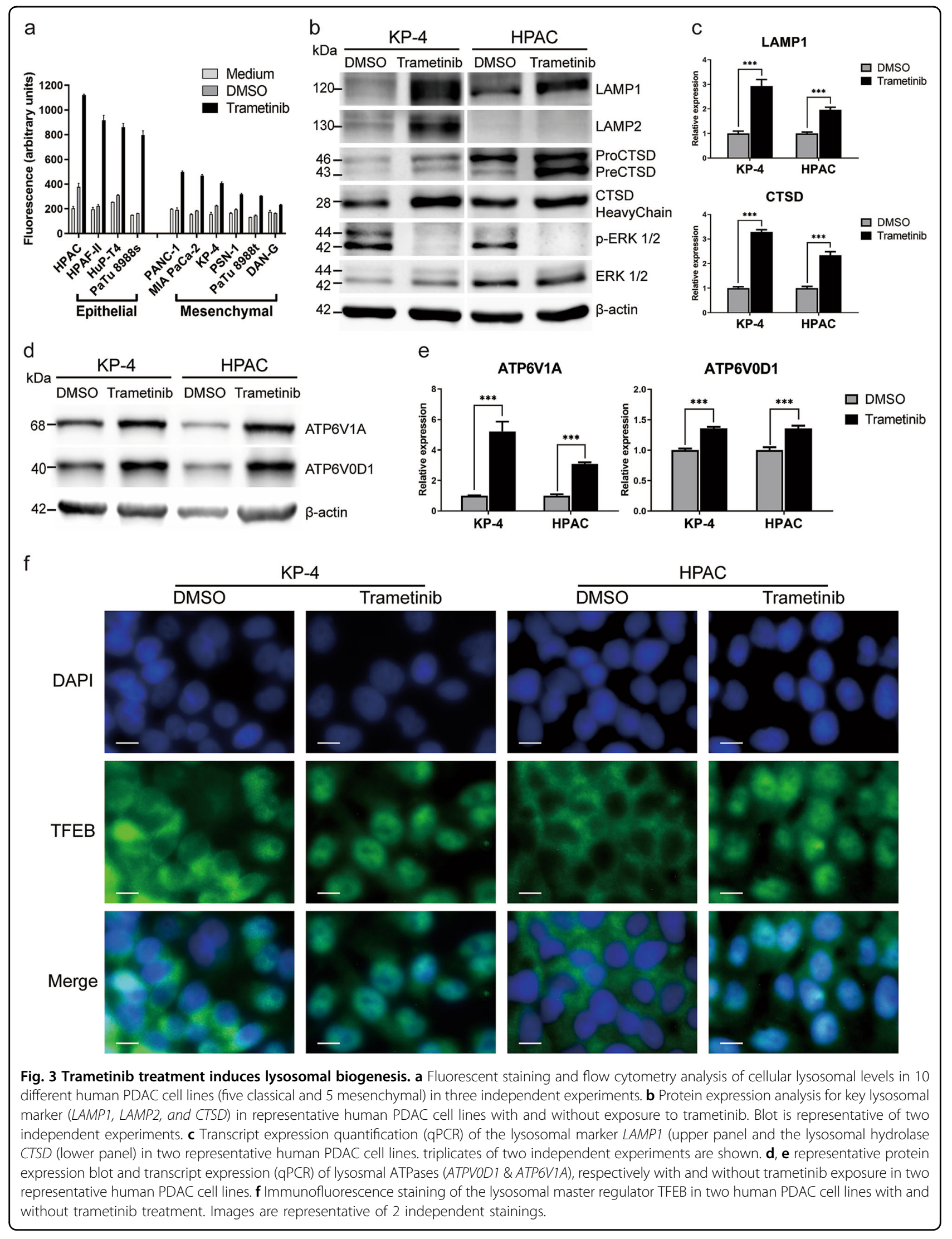


siRNA construct, TFEB knockdown was performed in 4 PDAC cell lines ( $2 \mathrm{cl}$ and $2 \mathrm{qm}$ ). In all 4 human PDAC cell lines, a knockdown of at least $60 \%$ was achievable (Fig. $4 \mathrm{a}$ ) and the protein expression was equally downregulated (Fig. 4b). Furthermore, the expression of direct TFEB target genes was significantly reduced (Fig. 4c and Supplementary Fig. 1b). As shown in Fig. 4d, downregulation of TFEB led to stabilized lysosomal levels upon MEKi treatment in both $\mathrm{cl}$ and $\mathrm{qm}$-type PDAC cell lines. Indeed, fluorescent lysosomal staining of the TFEB-depleted cells after trametinib treatment did not show any significant increase in lysosomal contents as was observed in wildtype cells or cells transfected with a non-targeting siRNA. Both transcript and protein levels of lysosomal markers LAMP1 and $L A M P 2$ as well as lysosomal-associated ATPases such as vATP6VOD1 and the hydrolase CTSD remained stable in the MEKi-treated group after TFEB knockdown compared with cell transfected with a non-targeting control (Fig. 4e, f).

\section{Trametinib accumulates in lysosomes and causes lysosomal exocytosis}

Weak base anticancer drugs can be trapped in the lysosomes by cation trapping ${ }^{16}$. We next wanted to determine if increased cellular lysosomal levels could lead to drug sequestration. To determine if trametinib undergoes lysosomal trapping and possible excretion, cells were treated with a single $\mathrm{IC}_{50}$ dose of trametinib and lysosomes were isolated to directly quantify the amount of trametinib within the isolated lysosomes. As shown in Supplementary Fig. 6, the isolated cellular fraction was highly enriched for the lysosomal marker $L A M P 2$ and the lysosomal vATPase ATP6VOD1. After sonication to lyse the lysosomes and liberate their content, the lysosomal content was assessed for the presence of trametinib by LC-MS. Fig. 5b and Supplementary Fig. $7 \mathrm{c}$ show the mass spectra of trametinib from the isolated lysosomes and the trametinib standard respectively, indicating the presence of the drug in these organelles. We then investigated if the sequestered drug was disposed of in the extracellular milieu. We collected cell culture supernatant from trametinib-treated cells and isolated the protein fraction by ultrafiltration. This fraction was analyzed by western blot as shown in Fig. 5a. In effect, there were higher levels of the lysosomal hydrolase CTSD and the ATPase ATP6VOD1 in cell culture supernatants from trametinib-treated cells compared with the vehicle control group. This suggest that lysosomal contents is being emptied in the extracellular milieu, although cell death could contribute partially as well.

\section{TFEB silencing sensitizes PDAC cells to MEK inhibition}

Given the role of TFEB in MEKi-induced lysosomal biogenesis and the observation that trametinib is sequestered into the lysosomes and potentially excreted by lysosomal exocytosis, we asked whether TFEB knockdown would sensitize the cells to MEK inhibition. Indeed, after TFEB knockdown all tested cell lines were more sensitive to trametinib treatment as seen in the corresponding $\mathrm{IC}_{50}$ values (Fig. $5 \mathrm{c}$ ). We performed a colony formation assay in MEKi-treated vs vehicle-treated TFEB knockdown and scramble cells. As shown in Fig. 5d, although TFEB knockdown affected the viability of the classical cell line HPAC in the absence of trametinib, no such difference was observed in the qm PDAC cell line KP4. However, upon trametinib treatment, cell viability was strongly affected in both cell lines, with a more pronounced effect observed in the TFEB knockdown group of the qm PDAC cell line KP4.

\section{MEK inhibition triggers lysosomal biogenesis in human PDAC in vivo}

Finally, we assessed whether MEKi-induced lysosomal biogenesis occurs in human PDAC in vivo. To test this hypothesis, we first performed gene expression analyses on human PDAC cell lines treated with single dose $\mathrm{IC}_{50}$ of trametinib or vehicle control. Additionally, using publicly available gene expression profiling data from human PDAC cell lines treated with a different MEK inhibitor (CI-1040) we performed Gene set enrichment analyses. In cell line treated with CI-1040, there was a strong enrichment in lysosomal gene set (Fig. 6a). Similarly, in cell line treated with the MEK inhibitor Trametinib, there was enrichment for lysosomal genesets, and the expression of core enriched genes increase with decreasine trametinib concentration (Fig. 6b). We then stained for the lysosome-specific marker LAMP1 in trametinib-treated human PDAC xenograft tissues. Human PDAC PDX were generated and treated as described previously ${ }^{23}$. As shown in Fig. 6c and Supplementary Fig. 7b, human PDAC PDX models treated with the MEK inhibitor trametinib showed a significantly high expression of the lysosomal membrane marker LAMP1 in the treated group compared with the vehicle group. The TFEB target gene ATP6V1A was equally highly expressed under MEKi treatment (Supplementary Fig. 7a).

\section{Discussion}

The action mode of targeted agents is generally predicted through complex computer assisted modeling, followed by biological validation and molecular imaging $^{24-26}$. However, intracellular drug interaction may also affect drug efficacy. In addition to adaptive resistance mechanisms such as drug efflux, activation of alternative oncogenic pathway, mutations development, and other factors such as selective drug sequestration may result in cellular unresponsiveness ${ }^{27-29}$. Lysosomal drug sequestration accounts for multi-drug resistance to several targeted therapeutic agents in cancer $^{30,31}$. 


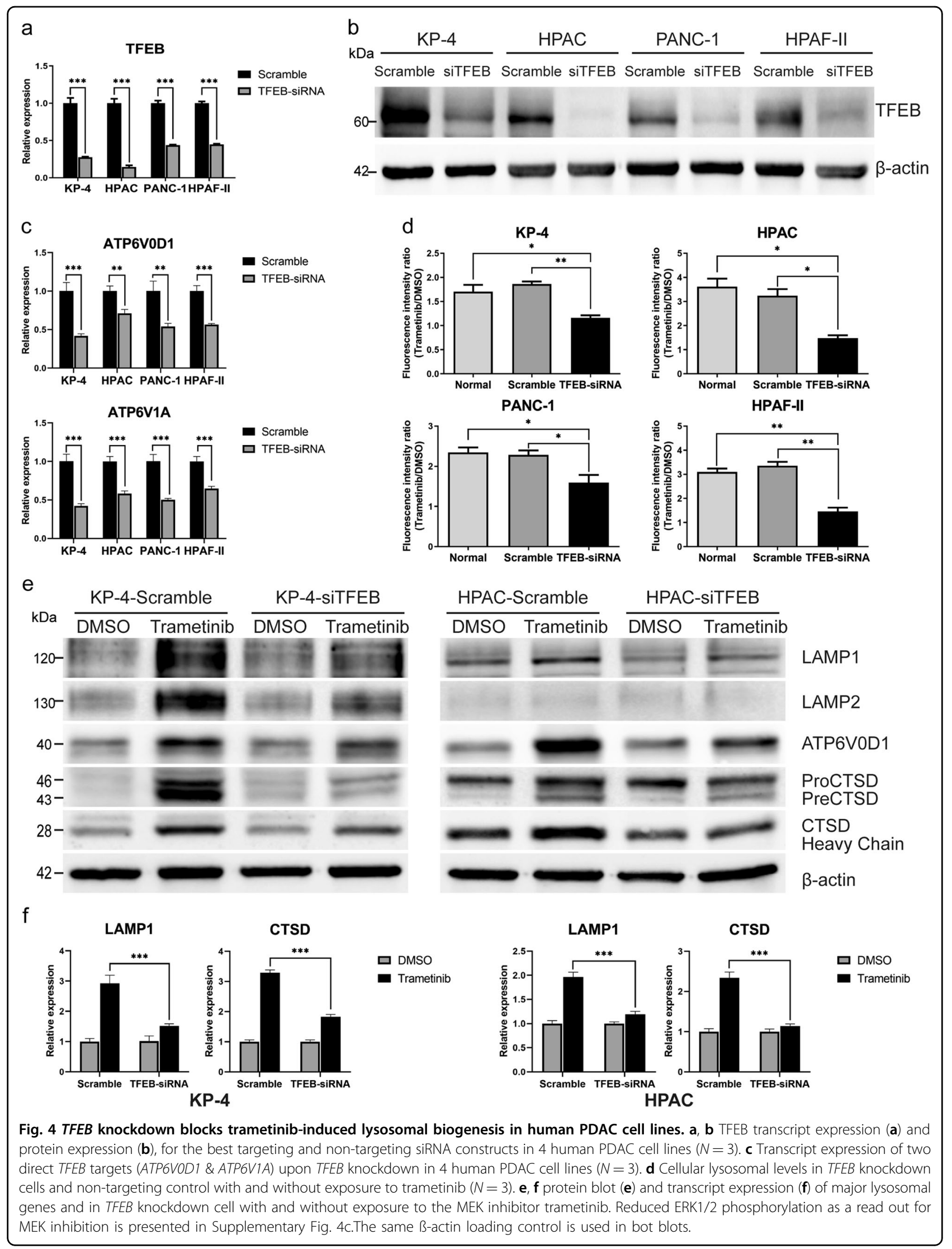




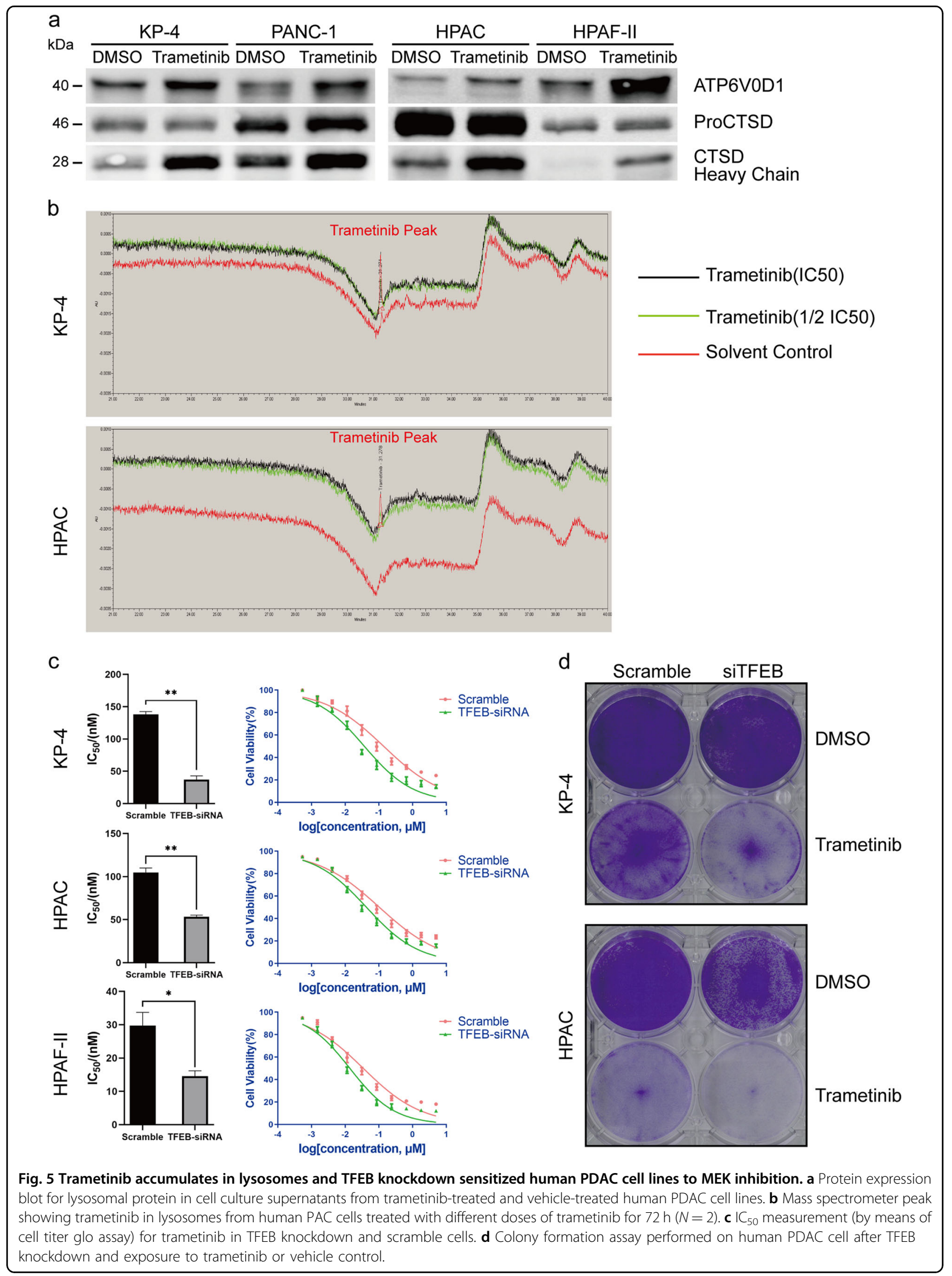




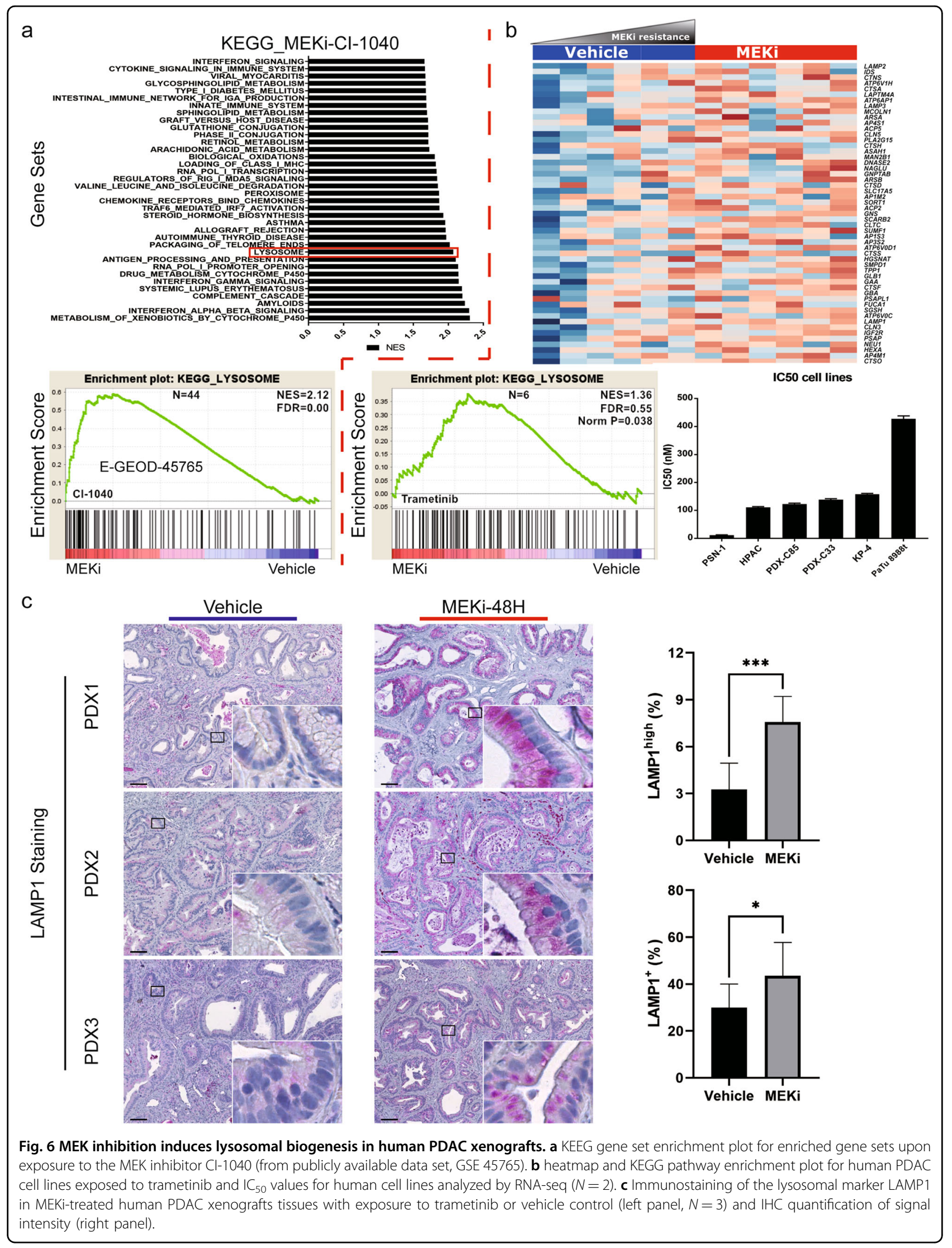


We investigated MEKi-induced lysosomal drug accumulation in vivo and in vitro using human and mouse PDAC models to understand if drug sequestration could partly explain resistance observed to very potent MEK inhibitors such as trametinib. Treatment of PDAC tumors and cells with different MEK inhibitors led to increased lysosomal content and a consequent upregulation of lysosomal makers and lysosome-associated genes. Additionally, nuclear translocation of TFEB was observed after MEK inhibition and a corresponding upregulation of its target genes. This may point to the fact that MEK inhibition in PDAC induce lysosomal biogenesis and foster lysosomal drug accumulation and subsequent exocytosis as previously hypothesized ${ }^{32}$. To verify this hypothesis, we isolated lysosomes from trametinib-treated cells and using mass spectrometry,we were able to detect the presence of trametinib in these organelle.

Lipophilic amines with a pKa between 6.5 and 11 tend to accumulate more in a concentration-dependent manner in lysosomes ${ }^{33}$. Additionally, some anticancer drugs such as Gefitinib and Lapatinib (with a pKa of 4.5 and 3.8, respectively) are also trapped in lysosomes ${ }^{34}$. Irrespective of their acid-base properties, cells may sequester certain xenobiotics into the lysosomes as a detox mechanism. In fact, lysosomal drug sequestration plays a protective role in brain cells where lysosomes trap drugs and deposit them in neutrophils by extrusion ${ }^{35}$. Malignant cancer cells may adopt such mechanisms to guard against therapeutic assaults. Indeed, lysosomal drug sequestration has been reported for other anticancer agents ${ }^{36}$ and is responsible, at least in part for drug resistance $e^{32,37,38}$.

In human PDAC cell lines, we observed a strong increase in lysosomal levels in clPDAC cells compared to qmPDAC cells. In qmPDAC cells, TFEB was already present in the nucleus in basal conditions, as opposed to clPDAC cells, in which nuclear TFEB was observed only upon MEKi treatment. The presence of nuclear TFEB in qmPDAC cells before treatment may suggest a pre-existing xenobiotic sequestration and extrusion mechanism that accounts for the high resistance capabilities observed in these cells. This hypothesis is strengthened by the observation that a mesenchymal or basal-like phenotype is associated with poor outcome both in pancreatic and breast tumors ${ }^{39,40}$. Additionally, siRNA-mediated depletion of TFEB led to a more pronounced sensitization of qmPDAC cells to MEK inhibition compared to clPDAC cells. In fact, a 3-fold increase in MEKi sensitivity was observed in qMPDAC cells as opposed to a 1.5-2-fold increase in clPDAC cells. Lysosomal drug trafficking may therefore be contributing at least partially to intrinsic and acquired resistance to MEK inhibition in PDAC. This is further supported by the observation of a linear relationship between MEKi sensitivity and transcript abundance of lysosome-associated genes in vehicle-treated PDAC cells.
Accumulation of MEK inhibitors in intracellular lysosomes was associated with increased levels of lysosomal enzymes in cell culture supernatants and the presence of MEK inhibitor in intracellular lysosomes. Previous studies have reported TFEB-mediated lysosomal biogenesis and exocytosis upon treatment with different anti-cancer drugs $^{16}$. Lysosomal targeting equally potentiates the activity of nintedanib in the treatment of non-small cell lung cancer ${ }^{27}$. Besides MEK inhibitor accumulation and exocytosis presented here, other targeted anticancer drugs such as tyrosine kinase inhibitors have also been reported to be accumulated in lysosomes ${ }^{31,41}$. Lysosomal drug accumulation and subsequent exocytosis may reduce the amount of bioavailable molecule at its target site thereby reducing its efficacy.

\section{Conclusion}

Taken together, our data provides evidence of lysosomal drug sequestration and exocytosis during MEK inhibition both in vivo and in vitro in PDAC. We also show, that qmPDAC cells are more dependent on TFEB for modulation of MEKi sensitivity and demonstrate the presence of the targeted agent, trametinib, inside the lysosomes, an observation that may partly explain resistance to trametinib in PDAC.

\section{Materials and methods \\ Cell culture and treatment}

PDAC cells were obtained from the American Type Culture Collection (ATCC). All Cells were cultured in Dulbecco's Modified Eagle's Medium (cat no. 41965062, Life Technologies, Darmstadt, Germany) supplemented with $10 \% \mathrm{FBS}$ and $1 \%$ pen-strep in a humidified incubator at $37^{\circ} \mathrm{C}$ with $5 \% \mathrm{CO}_{2}$. For short-term MEK inhibition, cells were treated with a single $\mathrm{IC}_{50}$ dose of trametinib (cat no. SYN-1170-M010, Biomol, Hamburg, Germany) for $72 / 48 \mathrm{~h}$ (human cell lines/mouse cell lines) or an equal volume of vehicle control (DMSO). Long-term MEK inhibition in mouse PDAC cell lines was achieved by chronic exposure to increasing doses of trametinib up to $100 \mathrm{x} \mathrm{IC}_{50}$. Four different siRNA targeting TFEB (Supplementary Table 1) were purchased from Qiagen. Transfections were performed using the HiPerFect transfection reagent (cat no. 301705, Qiagen, Hilden, Germany) according to the manufacturer's instructions and analyzed by western blotting and qPCR.

\section{Mice and treatments}

Ptfla ${ }^{\text {wt/cre }} ; K_{r a s}^{\text {wt/LSL-KrasG12D }} ; T p 53^{\text {fl/fl }}$ (CKP) mice developing spontaneous aggressive PDAC were generated as previously described ${ }^{42}$ and treated for $48 \mathrm{~h}$ with the MEK inhibitor refametinib ${ }^{43}$. Patient-derived xenografts were generated by subcutaneous transplantation of resected PDAC tumors or from purified circulating 
tumorigenic cancer stem cells in NOD/SCID/IL2y- mice and propagated in NMRI:nu/nu mice after engraftment ${ }^{23}$. After morphological and molecular characterization, animals were treated with a single dose of trametinib $(1 \mathrm{mg} / \mathrm{kg})$ as previously reported ${ }^{23}$.

\section{RNA sequencing and gene set enrichment analyses}

Total RNA was isolated using the Maxwell RSC simplyRNA Cells Kit (cat no. AS1390, Promega, Fitchburg, USA) following manufacturer's instruction. $100 \mathrm{ng}$ of total RNA was used for library preparation using the TruSeq Stranded mRNA (cat no. 20020595, Illumina, San Diego, USA) and subjected to $100 \mathrm{bp}$ paired end sequencing on a HiSeq 4000. After quality control with FastQC, sequencing reads were trimmed and mapped to the human genome (Genome 38) with STAR and features were quantified using htSeq count. Additionally, publicly available data from MEKi-treated PDAC cell lines was downloaded from the gene expression omnibus (GSE$45765)^{44}$.

Gene set enrichment analyses (GSEA) was performed using the Broad Institute algorithm with gene set permutation on the Hallmarks, KEGG, Reactome and Gene ontology gene sets. Significance cut off was set to a false discovery of $<0.05$ (Benjamini \& Hochberg).

\section{Lysosomal staining and flow cytometry}

For lysosomal staining, cells were grown to about 70\% confluence and then incubated with Cytopainter LysoGreen indicator reagent $(1: 5000$, cat no. ab176826, Cambridge Biomedical Campus, Cambridge, UK) for $1 \mathrm{~h}$ at $37^{\circ} \mathrm{C}$ with $5 \% \mathrm{CO}_{2}$. Cells were then washed twice in pre-warmed Hank's balanced salt solution (HBSS, cat no. 14175053, Life Technologies), trypsinized with 0.05\% trypsin EDTA (cat no. 25300054, Life Technologies) and resuspended in $1 \mathrm{x}$ phosphate buffered saline (PBS, cat no. 14190169, Life Technologies) for flow cytometry. Lysosomal levels were measured by quantifying the dye fluorescence in a Beckman Coulter Gallios flow cytometer (Beckman Coulter, Brea, USA).

\section{Immunohistochemical staining and quantification (IHC)}

Immunostaining was performed on $5 \mu \mathrm{M}$ sections of formalin-fixed paraffin-embedded tissues using the Dako REAL Alkaline Phosphatase Detection System (cat no. K500511-2, Dako, Santa Clara, USA). Sections were dewaxed on an automated dewaxing system (Leica ST5010 Autostainer XL, Leica Geosystems, Heerbrugg, Switzerland) and heat-induced epitope retrieval was performed using citrate buffer ( $10 \mathrm{mM}$ Sodium Citrate, $0.05 \%$ Tween $20, \mathrm{pH}=6$ ) for $15 \mathrm{~min}$ at $110^{\circ} \mathrm{C}$ in a pressure cooker. After blocking with serum-free protein blocking solution for $30 \mathrm{~min}$ (cat no. X0909, Dako), slides were incubated with a 1:250 dilution of primary antibodies at
$4{ }^{\circ} \mathrm{C}$ overnight. The slides were then washed in TBS-T buffer 3 times for $5 \mathrm{~min}$,incubated with secondary antibody for $30 \mathrm{~min}$ at room temperature, and then subjected to Fast Red chromogen development using permanent AP red kit (cat no. ZUC001-125, Zytomed Systems, Berlin, Germany). Slides were counterstained with hematoxylin, dehydrated, and mounted.

Slides were scanned and digitalized with a Zeiss Axio Scanner Z.1 (Carl Zeiss AG) at 2.5X and 10X magnification. The percentage of positive cells was quantified by Definiens software version 4.3.1 (Definiens AG) on the whole area of the pancreatic ductal cells per field. The total number of cells in each field was determined based on nuclei staining detected by the software.The average number of positive cells and high positive cells were expressed as a percentage of the total number of cells from each field.

\section{Immunofluorescence staining}

Cells were grown on 8-well cell culture chamber slides (cat no. 154534, Lab-Tek, Waltham, USA). 3 days after treatment with a single $\mathrm{IC}_{50}$ dose of trametinib or DMSO control (cat no. D2438, Sigma-Aldrich, St. Louis, USA), slides were washed with PBS 3 times and fixed in $4 \%$ paraformaldehyde in PBS for $15 \mathrm{~min}$. Slides were then washed with cold PBS 3 times for $5 \mathrm{~min}$ and permeabilized with $0.3 \%$ Triton-X100 in PBS for 20 min at room temperature. After washing as before, slides were blocked with 5\% BSA in PBS for 45 min. Cells were incubated with a 1:100 dilution of the corresponding primary antibodies (Supplementary Table 2) in $1 \%$ BSA at $4{ }^{\circ} \mathrm{C}$ overnight. Slides were washed and incubated with the secondary antibody (1:400; cat $\mathrm{N}^{\circ}$ SA5-10086, Thermo Scientific, Waltham, USA) for $1 \mathrm{~h}$ at room temperature in the dark. Afterwards, slides were washed 3 times with PBS, counterstained with DAPI (cat $\mathrm{N}^{\circ} \mathrm{H}-1200$, Vector Laboratories, Burlingame, USA) and scanned with a Zeiss Scanner (40x).

\section{Cell viability $\left(\mathrm{IC}_{50}\right)$ and colony formation assays}

Trametinib was dispensed in white flat bottom 96 well plates in triplicate in a logarithmic range from $0.0015 \mu \mathrm{M}$ to $5 \mu \mathrm{M}$, and 3000-4000 cells (cell line dependent optimal numbers, Supplementary Table 3) were seeded in the drug-containing plates using a multidrop (Multidrop Combi, Thermo Scientific). After $72 \mathrm{~h}$, an equal volume of CellTiter-Glo reagent (cat $\mathrm{N}^{\circ}$ G7573, Promega) was added, mixed vigorously by shaking for $2 \mathrm{~min}$ and incubated at room temperature for $10 \mathrm{~min}$. Luminescence signal was measured on the Spark Multimode Microplate Reader (Tecan Group, Männedorf, Switzerland). Triplicates were averaged,dose response curves were analyzed in GraphPad Prism version 8 and $\mathrm{IC}_{50}$ values were determined. 
For colony formation assays, cells were grown in 6well plates to a $30 \%$ confluency and treated with a single $\mathrm{IC}_{50}$ drug dose or vehicle control for $72 \mathrm{~h}$. Cells were then washed with PBS once at room temperature, fixed for $10 \mathrm{~min}$ in ice-cold methanol and then stained with crystal violet (cat no. C0775-25G, Sigma-Aldrich) for $30 \mathrm{~min}$. Plates were washed twice with water and dried for $24 \mathrm{~h}$.

\section{Secretome protein isolation}

Cells were cultured to $75-85 \%$ confluence in a T175 flask. Cell culture medium was then removed and cells were washed 3 times with PBS followed by 2 times wash with serum-free growth medium. Subsequently, cells were treated with a single $\mathrm{IC}_{50}$ dose of trametinib or vehicle control for $48 \mathrm{~h}$ in serum-free growth medium. The cell culture supernatant was then collected and centrifuged at $500 \mathrm{~g}$ for $5 \mathrm{~min}$. The resulting supernatant was concentrated using $3 \mathrm{kDa}$ molecular-weight cutoffs polyethersulfone membrane (cat no. PES-3K, Pierce Protein Concentrator, Thermo Scientific) by centrifugation at $3500 \mathrm{xg}$ in a swinging-bucket rotor at $4{ }^{\circ} \mathrm{C}$ for $3-4 \mathrm{~h}$ and stored at $-20^{\circ} \mathrm{C}$.

\section{Lysosomal isolation}

Lysosomal fractions were isolated from cultured cells by density gradient centrifugation in a Optima L-80 XP Ultracentrifuge $(35,000 \mathrm{rpm}, 2 \mathrm{~h})$ using the Lysosome Enrichment Kit for Tissue and Cultured Cells (cat no. 89839, Thermo Scientific), according to the manufacturer's protocol. The purity of the fractions was assessed by measuring expression of lysosome-specific protein by western blot. Accumulation of trametinib in lysosomes was analyzed by liquid chromatography coupled to mass spectrometry (LC-MS, Waters GmbH).

\section{Identification of trametinib in lysosomes}

Lysosomal Trametinib was analyzed with a RP-HPLC (Waters $\mathrm{GmbH}$, Eschborn, Germany). Analytes were separated on an EC250/4 Nucleodur C18 ISIS, $5 \mu \mathrm{m}$ (cat no. 760414.40) with pre-column CC8/4 (cat. no. 761310.40, Macherey-Nagel, Düren, Germany) thermostatted at $40{ }^{\circ} \mathrm{C}$. A gradient at a flow rate of $1.00 \mathrm{ml} / \mathrm{min}$ was achieved with mobile phase of $\mathrm{A}=$ formic acid $(0.1 \%)$, $\mathrm{B}=$ methanol, and $\mathrm{C}=$ acetonitrile, respectively. Split ratio between PDA- and MS-detector was set to 85/15 (PDA/MS, vol/vol). Mass spectrometry was performed in positive ion electrospray ionization mode. Masses for the detection of Trametinib were optimized after extraction from total ion chromatogram.

\section{Western blot analysis}

Proteins were extracted in 1x RIPA Buffer (cat no. 9806S, Cell Signaling Technologies, Danvers, USA) supplemented with $1 \mathrm{x}$ protease and phosphatase inhibitor cocktails (cat no. 5872, Cell Signaling technologies). Protein concentration was determined by BCA protein assay (cat no. 23225, Thermo Scientific). Samples were resolved on $8-10 \%$ SDS polyacrylamide gels and blotted on $0.2 \mu \mathrm{M}$ nitrocellulose membranes (cat no. 1704270, Bio-Rad, Hercules, USA). Membranes were blocked with $5 \%$ BSA (in TBS-T) at room temperature for $1 \mathrm{~h}$ on an orbital shaker and then incubated with primary antibodies diluted in blocking buffer at $4{ }^{\circ} \mathrm{C}$ overnight. Secondary antibodies were diluted in blocking buffer and incubated at room temperature for $1.5 \mathrm{~h}$. Membranes were developed with the SuperSignal Chemiluminescent (cat no. 34075, Thermo Scientific) and scanned with a ChemiDoc MP Imaging System (Bio-Rad).

\section{Real-time quantitative PCR}

Total RNA was extracted using the Maxwell RSC simplyRNA Cells Kit (cat no. AS1390, Promega) according to the manufacturer's instructions. cDNA was synthesized from $1 \mu \mathrm{g}$ of total RNA using SuperScript IV First-Strand Synthesis System (cat no. 18091200, Thermo Scientific). Transcript quantification was performed using a $1 \mathrm{x}$ sybrgreen mix in $12 \mu \mathrm{l}$ reactions on a Roche LightCycler 480 using Light Cycler 480 SYBR Green I Master Kit (cat no. 04887352001, Roche GmbH, Basel, Switzerland) Primer sequences used are listed in Supplementary Table 4.

\section{Acknowledgements \\ This work was partly funded by the Deutsche Forschungsgemeinschaft (DFG, German Research Foundation) - SO 1549/3-1 (KFO 337). \\ Author details \\ 'Division of Solid Tumor Translational Oncology, German Cancer Consortium (DKTK, partner site Essen) and German Cancer Research Center, DKFZ, Heidelberg, Germany, Division of Solid Tumor Translational Oncology, German Cancer Consortium (DKTK, partner site Essen) and German Cancer Research Center, DKFZ, Heidelberg, Germany, Essen, Germany. ${ }^{2}$ Institute for \\ Developmental Cancer Therapeutics, West German Cancer Center, University Hospital Essen, Essen, Germany, Heidelberg, Germany. ${ }^{3}$ Department of Hepatobiliary Surgery, the First Affiliated Hospital of Dalian Medical University, Dalian, Liaoning Province, China. ${ }^{4}$ Dept Med Oncol, West German Cancer Center, University Hospital Essen, Essen, Germany. ${ }^{5}$ nstitute for Cell Biology, University Hospital Essen, Essen, Germany. ${ }^{6}$ Translational Genomics in Solid Tumors, West German Cancer Center, University Hospital Essen, Essen, Germany. ${ }^{7}$ EPO - Experimental Pharmacology and Oncology GmbH Berlin- Buch, Berlin, Germany. ${ }^{8}$ Department of Molecular Gl-Oncology, Rurh University Bochum, Bochum, Germany}

Conflict of interest

The authors declare that they have no conflict of interest.

\section{Publisher's note}

Springer Nature remains neutral with regard to jurisdictional claims in published maps and institutional affiliations.

The online version of this article (https://doi.org/10.1038/s41420-020-0246-7) contains supplementary material, which is available to authorized users. 
Received: 22 October 2019 Revised: 20 February 2020 Accepted: 24 February 2020

Published online: 11 March 2020

\section{References}

1. Siegel, R. L., Miller, K. D. \& Jemal, A. Cancer statistics, 2016. CA Cancer J. Clin. 66, 7-30 (2016).

2. Cancer Genome Atlas Research Network. Electronic address aadhe, Cancer Genome Atlas Research N. Integrated genomic characterization of pancreatic ductal adenocarcinoma. Cancer Cell 32, 185-203 (2017). el13.

3. Aung, K. L. et al. Genomics-driven precision medicine for advanced pancreatic cancer: early results from the COMPASS trial. Clin. Cancer Res. 24, 1344-1354 (2018).

4. Thomas, R. K. et al. High-throughput oncogene mutation profiling in human cancer. Nat. Genet. 39, 347-351 (2007).

5. Eser, S., Schnieke, A., Schneider, G. \& Saur, D. Oncogenic KRAS signalling in pancreatic cancer. Br. J. Cancer 111, 817-822 (2014).

6. Eser, S. et al. Selective requirement of PIJK/PDK1 signaling for Kras oncogenedriven pancreatic cell plasticity and cancer. Cancer Cell 23, 406-420 (2013).

7. Roberts, P. J. \& Der, C. J. Targeting the Raf-MEK-ERK mitogen-activated protein kinase cascade for the treatment of cancer. Oncogene 26, 3291-3310 (2007)

8. Sun, C. et al. Intrinsic resistance to MEK inhibition in KRAS mutant lung and colon cancer through transcriptional induction of ERBB3. Cell Rep. 7, 86-93 (2014).

9. Wicki, A. et al. Acquired resistance to clinical cancer therapy: a twist in physiological signaling. Physiol. Rev. 96, 805-829 (2016).

10. Caunt, C. J., Sale, M. J., Smith, P. D. \& Cook, S. J. MEK1 and MEK2 inhibitors and cancer therapy: the long and winding road. Nat. Rev. Cancer 15, 577-592 (2015).

11. Infante, J. R. et al. A randomised, double-blind, placebo-controlled trial of trametinib, an oral MEK inhibitor, in combination with gemcitabine for patients with untreated metastatic adenocarcinoma of the pancreas. Eur. J. Cancer 50, 2072-2081 (2014).

12. Maiti, A. et al. Phase II trial of MEK inhibitor binimetinib (MEK162) in RASmutant acute myeloid leukemia. Clin. Lymphoma Myeloma Leuk. 19, 142-148 (2019). e141.

13. Ragon, B. K. et al. Oral MEK $1 / 2$ inhibitor trametinib in combination with AKT inhibitor GSK2141795 in patients with acute myeloid leukemia with RAS mutations: a phase II study. Clin. Lymphoma Myeloma Leuk. 19, 431-440.e13 (2019).

14. Lu, H. et al. SHP2 inhibition overcomes RTK-mediated pathway re-activation in KRAS mutant tumors treated with MEK inhibitors. Mol. Cancer Ther. 18, 1323-1334 (2019).

15. Fedele, C. et al. SHP2 inhibition prevents adaptive resistance to MEK inhibitors in multiple cancer models. Cancer Discov. 8, 1237-1249 (2018).

16. Zhitomirsky, B. \& Assaraf, Y. G. Lysosomal accumulation of anticancer drugs triggers lysosomal exocytosis. Oncotarget 8, 45117-45132 (2017).

17. Zhitomirsky, B. \& Assaraf, Y. G. Lysosomal sequestration of hydrophobic weak base chemotherapeutics triggers lysosomal biogenesis and lysosomedependent cancer multidrug resistance. Oncotarget 6, 1143-1156 (2015).

18. Pena-Llopis, S. et al. Regulation of TFEB and V-ATPases by mTORC1. EMBO J. 30, 3242-3258 (2011).

19. Kinsey, C. G. et al. Publisher correction: protective autophagy elicited by RAF$>$ MEK $\rightarrow$ ERK inhibition suggests a treatment strategy for RAS-driven cancers. Nat. Med. 25, 861 (2019).

20. Jin, J., Guo, Q., Xie, J., Jin, D. \& Zhu, Y. Combination of MEK inhibitor and the JAK2-STAT3 pathway inhibition for the therapy of colon cancer. Pathol. Oncol. Res. 25, 769-775 (2019).

21. Merchant, M. et al. Correction: Combined MEK and ERK inhibition overcomes therapy-mediated pathway reactivation in RAS mutant tumors. PLOS ONE 13, e0192059 (2018).

22. Collisson, E. A. et al. Subtypes of pancreatic ductal adenocarcinoma and their differing responses to therapy. Nat. Med. 17, 500-503 (2011).
23. Behrens, D. et al. Preclinical evaluation of novel treatment strategies in patientderived xenograft (PDX) models of pancreatic cancer. Eur. J. Cancer 103, E101-E101 (2018).

24. Zhao, F. L., Yang, G. H., Xiang, S., Gao, D. D. \& Zeng, C. In silico analysis of the effect of mutation on epidermal growth factor receptor in non-small-cell lung carcinoma: from mutational analysis to drug designing. J. Biomol. Struct. Dyn. 35, 427-434 (2017).

25. Ung, M. H., Varn, F. S. \& Cheng, C. In silico frameworks for systematic preclinical screening of potential anti-leukemia therapeutics. Expert Opin. Drug Discov. 11, 1213-1222 (2016).

26. Hossam, M., Lasheen, D. S. \& Abouzid, K. A. Covalent EGFR inhibitors: binding mechanisms, synthetic approaches, and clinical profiles. Arch. Pharm. Weinheim) 349, 573-593 (2016).

27. Englinger, B. et al. Intrinsic fluorescence of the clinically approved multikinase inhibitor nintedanib reveals lysosomal sequestration as resistance mechanism in FGFR-driven lung cancer. J. Exp. Clin. Cancer Res. 36, 122 (2017).

28. Zhitomirsky, B. \& Assaraf, Y. G. Lysosomes as mediators of drug resistance in cancer. Drug Resist. Update 24, 23-33 (2016).

29. Lu, S. Y., Sung, T., Lin, N. W., Abraham, R. T. \& Jessen, B. A. Lysosomal adaptation: how cells respond to lysosomotropic compounds. PLOS ONE 12 e0173771 (2017).

30. de Klerk, D. J., Honeywell, R. J., Jansen, G. \& Peters, G. J. Transporter and lysosomal mediated (multi)drug resistance to tyrosine kinase inhibitors and potential strategies to overcome resistance. Cancers (Basel) 10, 503 (2018).

31. Englinger, B. et al. Lysosomal sequestration impairs the activity of the preclinical FGFR inhibitor PD173074. Cells 7, 259 (2018).

32. Azijli, K., Gotink, K. J. \& Verheul, H. M. The potential role of lysosomal sequestration in sunitinib resistance of renal cell cancer. J. Kidney Cancer VHL $\mathbf{2}$, 195-203 (2015).

33. Kazmi, F. et al. Lysosomal sequestration (trapping) of lipophilic amine (cationic amphiphilic) drugs in immortalized human hepatocytes (Fa2N-4 cells). Drug Metab. Dispos. 41, 897-905 (2013).

34. Nadanaciva, S. et al. A high content screening assay for identifying lysosomotropic compounds. Toxicol. Vitr. 25, 715-723 (2011).

35. Noack, A. et al. Mechanism of drug extrusion by brain endothelial cells via lysosomal drug trapping and disposal by neutrophils. Proc. Natl. Acad. Sci. USA 115, E9590-E9599 (2018).

36. Zhitomirsky, B. et al. Lysosomotropic drugs activate TFEB via lysosomal membrane fluidization and consequent inhibition of mTORC1 activity. Cell Death Dis. 9, 1191 (2018).

37. Guo, B., Tam, A., Santi, S. A. \& Parissenti, A. M. Role of autophagy and lysosomal drug sequestration in acquired resistance to doxorubicin in MCF-7 cells. BMC Cancer 16, 762 (2016).

38. Gotink, K. J. et al. Lysosomal sequestration of sunitinib: a novel mechanism of drug resistance (vol 17, pg 7337, 2011). Clin. Cancer Res. 18, 318-318 (2012).

39. Milioli, H. H., Tishchenko, I., Riveros, C., Berretta, R. \& Moscato, P. Basal-like breast cancer: molecular profiles, clinical features and survival outcomes. BMC Med. Genomics 10, 19 (2017).

40. Birnbaum, D. J., Finetti, P., Birnbaum, D., Mamessier, E. \& Bertucci, F. Validation and comparison of the molecular classifications of pancreatic carcinomas. Mol. Cancer 16, 168 (2017).

41. Fu, D. et al. Imaging the intracellular distribution of tyrosine kinase inhibitors in living cells with quantitative hyperspectral stimulated Raman scattering. Nat. Chem. 6, 615-623 (2014).

42. Mazur, P. K. et al. Combined inhibition of BET family proteins and histone deacetylases as a potential epigenetics-based therapy for pancreatic ductal adenocarcinoma. Nat. Med. 21, 1163 (2015).

43. Trajkovic-Arsic, M. et al. Apparent diffusion coefficient (ADC) predicts therapy response in pancreatic ductal adenocarcinoma. Sci. Rep. 7, 17038 (2017).

44. Gysin, S., Paquette, J. \& McMahon, M. Analysis of mRNA profiles after MEK1/2 inhibition in human pancreatic cancer cell lines reveals pathways involved in drug sensitivity. Mol. Cancer Res. 10, 1607-1619 (2012). 